\title{
Programing Performance of Silk Fibroin Superstrong Scaffolds by Mesoscopic Regulation among Hierarchical Structures
}

\section{Supporting information}

Yifan Zhang ${ }^{1,2,3}$, Huang $\mathrm{Tu}^{2,3}$, Ronghui $\mathrm{Wu}^{1,2,3}$, Aniruddha Patil ${ }^{3}$, Chen $\mathrm{Hou}^{3}$, Zaifu $\operatorname{Lin}^{3}$, Zhaohui Meng ${ }^{3}$, Liyun $\mathrm{Ma}^{2,3}$, Rui $\mathrm{Yu}^{3 *}$, Weidong $\mathrm{Yu}^{2 *}$, and Xiang Yang Liu ${ }^{1,3 *}$ ${ }^{1}$ Department of Physics, Faculty of Science, National University of Singapore, Singapore 117542, Singapore

${ }^{2}$ Key Laboratory of Textile Science \& Technology, Ministry of Education, College of Textiles, Donghua University, Shanghai 201620, China

${ }^{3}$ Research Institution for Biomimetics and Soft Matter, Fujian Provincial Key Laboratory for Soft Functional Materials Research, College of Physical Science and Technology, Jiujiang Research Institute, Xiamen University, Xiamen 361005, China E-mail:phyliuxy@nus.edu.sg;wdyu@dhu.edu.cn;yurui@xmu.edu.cn 
SI 1. The measurement principle of using THT to label $\beta$-structure is shown in the Figure S1. Under the excitation of $342 \mathrm{~nm}$, the curve displays a strong emission peak at $456 \mathrm{~nm}$, and under the excitation of $442 \mathrm{~nm}$, the curve obtained a weak peak around 480nm(Figure S1a). Therefore, when measuring the $\beta$-structure, the selected $442 \mathrm{~nm}$ excitation will not cause too much interference to the signal. Figure S1b hints at the response of THT to different contents of $\beta$-sheets, while the more $\beta$-sheets, the stronger the peak at $482 \mathrm{~nm}[1]$. Therefore, THT can quantitatively analyze the relative content of $\beta$-sheets structure in the solution.

(a)

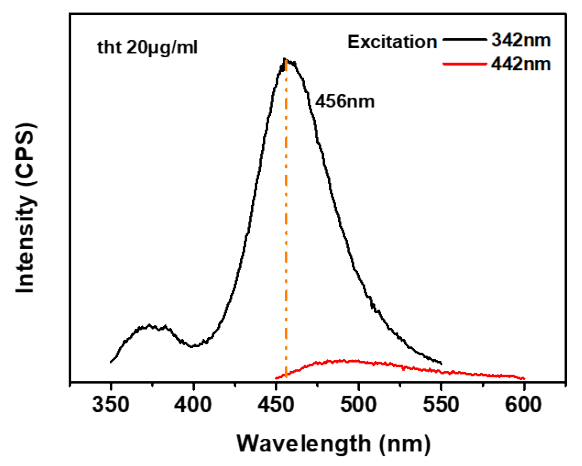

(b)

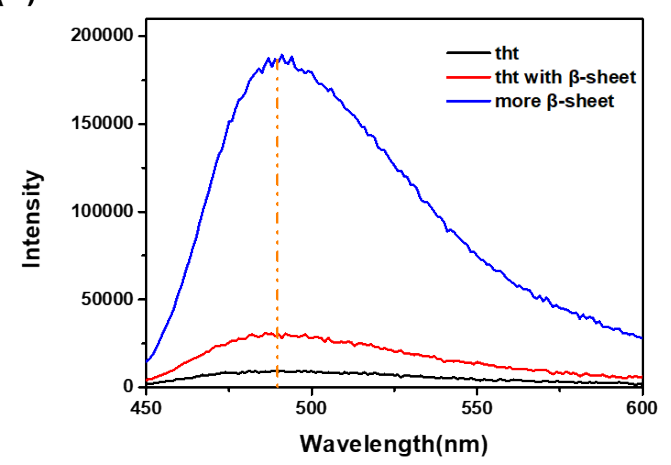

Figure S1. (a)Fluorescence spectra of ThT; (b) Fluorescence spectra of free and $\beta$-bound thioflavin (ThT). 
SI 2. The CD spectrum of the follow-up observation in the secondary structure of SF solution is shown in Figure $\mathrm{S} 2$. The content of the $\beta$-sheets is read through the absolute value of its characteristic peak intensity at $217 \mathrm{~nm}[2]$.

(a)

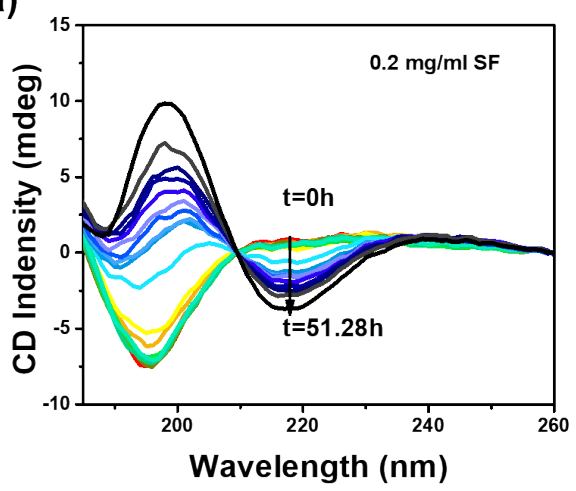

(b)

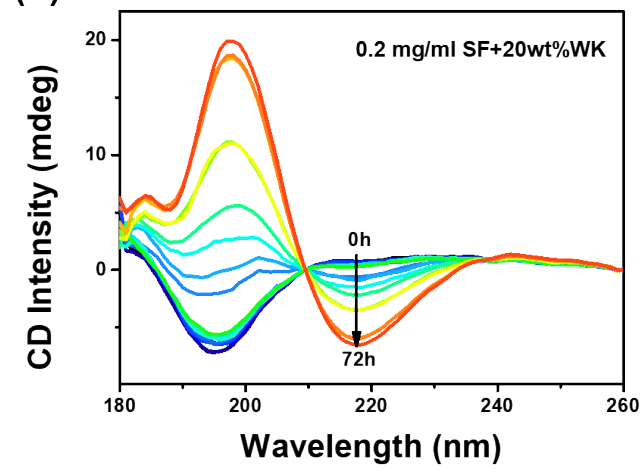

S2. (a) The change process of CD spectrum curve during the formation of $\beta$-sheet in neat WK solution; (b) The change process of CD spectrum curve during the formation of $\beta$-sheet in SF/WK solution 
SI 3. To further study the conformational changes of SF/WK chains during gelation, the structural transitions of freeze dried WK40 $\mathrm{wt} \%$ composites at $0 \mathrm{~h}, 1 \mathrm{~h}, 2 \mathrm{~h}$ and $4 \mathrm{~h}$ are determined by Fourier Transform Infrared Spectroscopy (FTIR) and X-ray Diffraction. Figure S3a shows FTIR spectral changes of SF/WK composites at different stages of gelation in amide I region which can be used as an indicator for evaluating the secondary structure of protein due to its small shift. As can be seen, the peak shifts from 1648 to $1630 \mathrm{~cm}^{-1}$ with increasing time in the gelation process[3]. This transition reveals an increased fraction of peak components in the range of $1628-1637 \mathrm{~cm}^{-1}$ which is owing to the $\beta$-sheet structure and the correspondingly decreased fraction at 1647 $1655 \mathrm{~cm}^{-1}$, owing to the random coil structure of protein[4]. The detailed calculation (Sa3) reflects that $\beta$-sheet content increases from $19 \%$ to $40 \%$ and shows $\mathrm{S}$-shaped curve as rheology curve of SF/WK composites displays, whereas the $\alpha$-helix content maintains around $10 \%$ without significant change, indicating that $\beta$-structural transitions mainly occur in the gelation process. The structural changes are further confirmed by XRD results (Figure Sa2), showing a great increase of $\beta$-crystal fraction (Silk II ) characterized by the signal density of diffraction peaks at $20.2^{\circ}$ and $24.7^{\circ}[5]$. From the quantitative analysis in Figure Sa3, it can be seen that the rise of $\beta$-crystals slower than $\beta$-sheets, implying that not all of the $\beta$-sheets stack into $\beta$-crystals. However, the similar increasing trend of $\beta$-crystal content with $\mathrm{G}^{\prime}$ and $\beta$-sheets indicates that the crystallization process is coincident with formation of the nanofibril networks. In a word, the gelation process is associated with the growth of nanofibrils and formation of $\beta$-structures, and the nanofibril networks are originated from stacking $\beta$-sheets into 
$\beta$-crystallites.
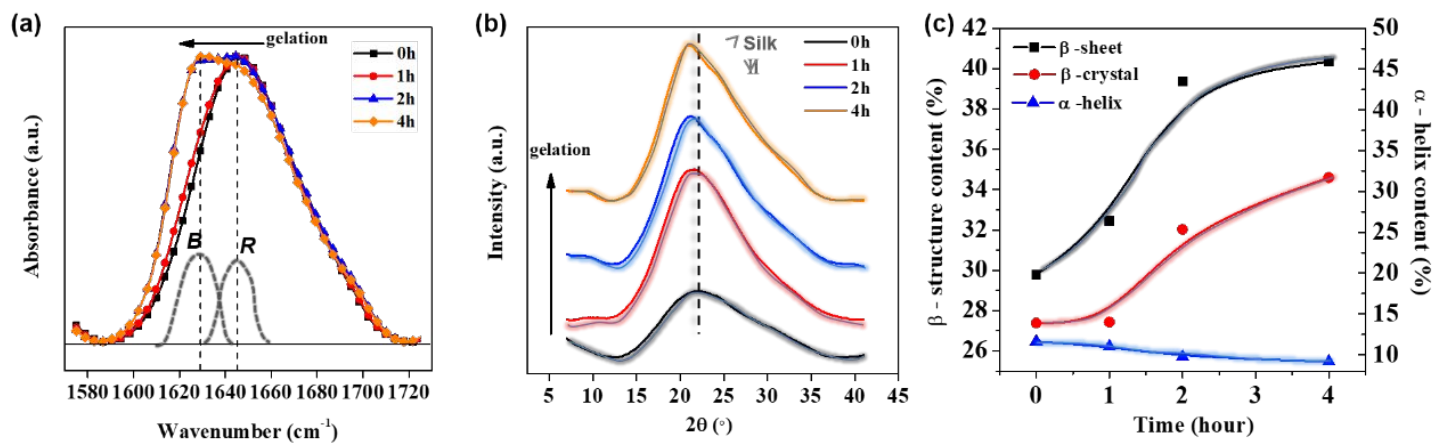

Figure S3. (a) FTIR spectra of freeze-dried SF/WK hydrogels in amide I region, which redshift from random coil (R) to $\beta$-sheet (B) during gelation process. (b) XRD results of freeze-dried SF/WK hydrogels indicate the variation of crystalline structures( Silk II) by the sharping crystalline peak around $2 \theta=20.2^{\circ}$ and the emergence of a new one at $2 \theta=24.8^{\circ}$. (c) Quantitative analysis for the changes in secondary structure fraction of freeze-dried SF/WK hydrogels during gelation. 
SI 4. The FTIR spectra of the scaffolds with different silk fibroin concentrations amide I region are shown in Figure S4a, which implies that the effect of WK on the secondary structure of the composite scaffold is much greater than the SF concentration. Therefore, when we discussing the interaction between WK and SF, the effect of SF concentration is neglected. The amide I band is deconvolved with Gaussian functions to determine the fraction of each secondary structure using the method of Fourier self-deconvolution (FSD)(Figure S4b).

(a)

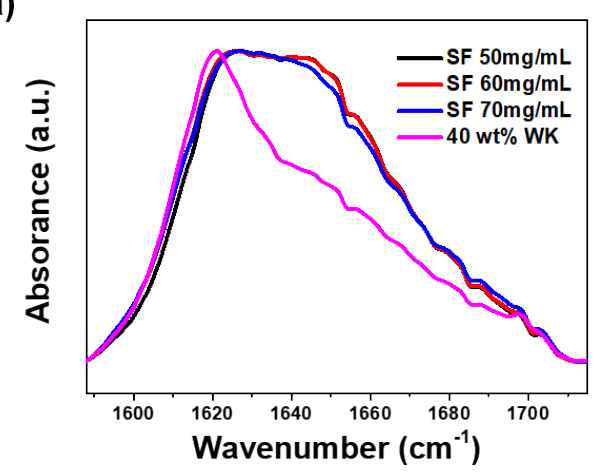

(b)

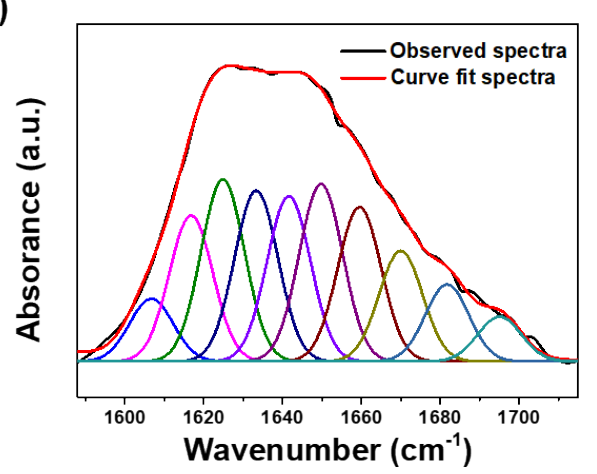

Figure S4. (a) The influence of the silk fibroin concentration on the secondary structure of the scaffold, which is not obvious enough compared with the scaffold containing keratin. (b) FTIR analysis of the secondary structure for SF scaffold sample prepared by casting $50 \mathrm{mg} / \mathrm{mL}$ SF solution with Gaussian distribution.

\section{Reference}

[1] LeVine III H. Quantification of $\beta$-sheet amyloid fibril structures with thioflavin T. Methods in Enzymology 1999. p. 274-84.

[2] Qiu W, Patil A, Hu F, Liu XY. Hierarchical Structure of Silk Materials Versus Mechanical Performance and Mesoscopic Engineering Principles. Small. 2019;15:e1903948.

[3] Du N, Liu XY, Narayanan J, Li LA, Lim MLM, Li DQ. Design of superior spider silk: From nanostructure to mechanical properties. Biophys J. 2006;91:4528-35. 
[4] Hu X, Kaplan D, Cebe P. Determining Beta-Sheet Crystallinity in Fibrous Proteins by Thermal Analysis and Infrared Spectroscopy. Macromolecules. 2006;39:6161-70.

[5] Jin HJ, Park J, Karageorgiou V, Kim UJ, Valluzzi R, Kaplan DL. Water-stable silk films with reduced beta-sheet content. Adv Funct Mater. 2005;15:1241-7. 